Interfaces and Free Boundaries 13 (2011), 57-72

DOI $10.4171 / \mathrm{IFB} / 248$

\title{
Analysis and simulation of debonding membranes
}

\author{
IOANA DURUS \\ Institut Elie Cartan Nancy, UMR 7502 CNRS, B.P. 239, 54506 Vandouvre-lès-Nancy, France \\ E-mail:ioanadurus@yahoo.com,durus@iecn.u-nancy.fr \\ ANNE LUX \\ LMAM CNRS UMR 7122, Université de Metz, Ile du Saulcy,57045 Metz, France \\ E-mail:lux@univ-metz.fr,anne.lux@laposte.net
}

[Received 23 March 2009 and in revised form 14 November 2010]

\begin{abstract}
The paper deals with the numerical analysis and simulation of debonding membranes in the framework of quasistatic processes modeled by minimizing movements. Depending on parameters of the model, the "support" of the membrane is a quasi-open set (i.e. the membrane is debonded or glued) or a capacitary measure (i.e. fractions of glue remain active). Time discretization is associated to gradient methods based on shape (measure) derivative and genetic shape (measure) optimization.
\end{abstract}

\section{Introduction}

We are concerned with the model of debonding membranes introduced by Bucur, Buttazzo and Lux in [6]. The debonding process is seen as a quasistatic evolution in the framework of minimizing movements governed by energy balance. Our goal is to propose a numerical simulation of debonding membranes subjected to an increasing force. Time discretization is associated to energy optimization using local algorithms, but also evolutionary algorithms. In fact, at each discrete time step of the minimizing movement process, we have to solve a global shape (or measure) optimization problem. Consequently, genetic algorithms are used in order to find (almost) global minimizers, and local algorithms based on shape derivative are used to model local deformations.

Since the merits and drawbacks of the model are discussed in [6], here we only briefly recall the model of quasistatic evolution of debonding membranes, and concentrate on numerical difficulties. We will also highlight the differences between the two situations we consider: when membranes are quasi-open sets or measures. In each case we implement two different algorithms. The first one is based on local deformation of sets or measures: it is a gradient method for capacitary measures (see for instance [14]) and a level-set/shape derivative method in the case of quasi-open sets (see for instance [1] and [22]). As the methods are local, we can only expect to find local minimizers, which from a mechanical point of view may be consistent with reality.

To be in agreement with the theoretical model and find (almost) global minimizers at each time step, we will also implement an evolutionary strategy method (detailed in [17], [21]) which has the advantage of having low sensitivity to initial estimates and the ability to escape local minima. We will give some details of this algorithm and present several numerical examples. For this purpose, we rely on a new method of generation of shapes and measures based on Fourier series ([7]). In order to validate our algorithms, in particular to capture jumps, we simulate the case of a radial membrane which was solved analytically in [6]. It turns out that our mixed algorithm provides a quite accurate simulation and, in particular, it finds jumps. We also compare global 
shape optimization results obtained by level set methods with the genetic algorithms. Tested on the example of radial debonding, for which the analytic solution is known, the global minimization algorithm based on the level set method gives good results only for particular initial states.

\section{Model of quasistatic evolution}

The main idea is to introduce a balance of energy between the potential energy of the membrane subjected to a debonding force $f$ and the energy required to debond it. The rule that governs the evolution of the membrane is energetic: the membrane has to be as stable as possible interplaying with the balance of energy.

The theoretical setting is not developed here; one should refer to [6] for a complete study. Let $D$ be a bounded open set (the support of the membrane) in $\mathbb{R}^{2}$ and $f \in L^{\infty}\left([0, T], L^{2}(D)\right)$ be the debonding force. We consider the debonding process as a quasistatic evolution, which means that for each time $t$ the membrane is in an equilibrium configuration. For our case, we first assume that the equilibrium of the membrane is determined by the following partial differential equation:

$$
\left\{\begin{array}{l}
-\Delta u+\mu u=f \quad \text { in } D \\
u \in H_{0}^{1}(D) \cap L^{2}(D, \mu),
\end{array}\right.
$$

where the state function $u$ in the Sobolev space $H_{0}^{1}(D)$ measures the vertical displacement of the membrane, and the nonnegative measure $\mu$ models the membrane together with the "active" fractions of glue. The case $\mu=0$ represents the complete debonding, while $\mu=+\infty$ represents the perfect adhesion of the membrane.

In our framework, $\mu$ varies in the class $\mathcal{M}_{0}$ of all nonnegative Borel measures on $D$, possibly taking the value $+\infty$, which are of capacitary type, that is, they vanish on all sets of capacity zero (for more details about these measures see for instance [8], [4]).

The energy of a membrane subjected to the debonding force $f$ and such that its adhesion is characterized by $\mu$ is given by

$$
E(\mu, f)=\min \left\{\frac{1}{2} \int_{D}|\nabla u|^{2} \mathrm{~d} x+\frac{1}{2} \int_{D} u^{2} \mathrm{~d} \mu-\int_{D} f u \mathrm{~d} x: u \in H_{0}^{1}(D) \cap L^{2}(D, \mu)\right\} .
$$

The energy required to debond the membrane is introduced in the following:

Definition 2.1 A dissipation distance on $\mathcal{M}_{0}$ is a mapping $\mathcal{D}: \mathcal{M}_{0} \times \mathcal{M}_{0} \rightarrow[0,+\infty]$ satisfying the following conditions:

(i) $\mathcal{D}(\mu, \mu)=0$ for every $\mu \in \mathcal{M}_{0}$;

(ii) $\mathcal{D}\left(\mu_{1}, \mu_{3}\right) \leqslant \mathcal{D}\left(\mu_{1}, \mu_{2}\right)+\mathcal{D}\left(\mu_{2}, \mu_{3}\right)$ for every $\mu_{1}, \mu_{2}, \mu_{3} \in \mathcal{M}_{0}$;

(iii) $\mu_{2} \npreceq \mu_{1} \Rightarrow \mathcal{D}\left(\mu_{1}, \mu_{2}\right)=+\infty$, where $\mu_{1} \preceq \mu_{2}$ denotes that $\int_{D} u^{2} \mathrm{~d} \mu_{1} \leqslant \int_{D} u^{2} \mathrm{~d} \mu_{2}$ for all $u \in H_{0}^{1}(D)$.

The irreversibility in the evolution of membranes is contained in condition (iii). The evolution of the membrane will be determined by the fact that the membrane will "search" an equilibrium between its energy and dissipation.

Given a debonding force $f(t, x) \geqslant 0$, we may now define the time discretization scheme of quasistatic evolution as follows:

- fix a time step $\epsilon>0$ and consider the discretized time $t_{k}^{\epsilon}=\epsilon k$ for $k \in \mathbb{N}$;

- start from an initial configuration $\mu_{0} \in \mathcal{M}_{0}$, so that $\mu_{\epsilon}(0)=\mu_{0}$; 
- define $\mu_{\epsilon}\left(t_{k}^{\epsilon}\right)$ iteratively, by taking $\mu_{\epsilon}\left(t_{k+1}^{\epsilon}\right)$ as the solution of the minimum problem

$$
\min \left\{E\left(\mu, f\left(t_{k+1}^{\epsilon}\right)\right)+\mathcal{D}\left(\mu, \mu\left(t_{k}^{\epsilon}\right)\right)\right\} ;
$$

- passing to the limit as $\epsilon \rightarrow 0$ we obtain a mapping $t \mapsto \mu(t)$ that is the generalized minimizing movement scheme.

Let us recall the definition of generalized minimizing movements, first introduced by De Giorgi in [13]. Consider a topological space $\mathcal{S}$, or more generally a set $\mathcal{S}$ endowed with a convergence structure, and a functional

$$
[0, T] \times \mathcal{S} \times \mathcal{S} \ni(t, v, w) \mapsto \mathcal{F}(t, v, w) \in \overline{\mathbb{R}} .
$$

For every fixed $\epsilon>0$, the Euler scheme with time step $\epsilon$ and initial condition $u_{0} \in \mathcal{S}$ consists in constructing a function $u_{\epsilon}(t)=w([t / \epsilon])$, where [.] stands for the integer part function, in the following way:

$$
w(0)=u_{0}, \quad w(n+1) \in \operatorname{Argmin}\{\mathcal{F}((n+1) \epsilon, \cdot, w(n))\} .
$$

Definition 2.2 We say that $u:[0, T] \rightarrow \mathcal{S}$ is a generalized minimizing movement associated to $\mathcal{F}$ with initial condition $u_{0}$, and we write $u \in \operatorname{GMM}\left(\mathcal{F}, \mathcal{S}, u_{0}\right)$, if there exists a sequence $\epsilon_{n} \rightarrow 0^{+}$ such that for any $t \in[0, T], u_{\epsilon_{n}}(t) \rightarrow u(t)$ in $\mathcal{S}$.

A rate independent model defined in a general framework by Mielke [18] (see also Mainik and Mielke [16]) and adapted to our problem is introduced in the next definition.

Definition 2.3 A couple $(u, \mu):[0, T] \rightarrow H_{0}^{1}(D) \times \mathcal{M}_{0}$ is called a solution of the rateindependent problem associated with the energy $E$ and the dissipation distance $\mathcal{D}$ if the following relations hold:

(S) Stability: For all $t \in[0, T]$ and all $\tilde{\mu} \in \mathcal{M}_{0}$ we have

$$
E(\mu(t), f(t)) \leqslant E(\tilde{\mu}, f(t))+\mathcal{D}(\tilde{\mu}, \mu(t)) ;
$$

(E) Energy inequality: For all $s, t \in[0, T]$ with $s<t$ we have

$$
E(\mu(t), f(t))+\operatorname{Diss}_{\mathcal{D}}(\mu,[s, t]) \leqslant E(\mu(s), f(s))+\int_{s}^{t}\left\langle\partial_{f} E(\mu(\tau), f(\tau)), \dot{f}(\tau)\right\rangle \mathrm{d} \tau
$$

where

$$
\operatorname{Diss}_{\mathcal{D}}(\mu,[s, t])=\sup _{N \in \mathbb{N}, s=t_{0}<\cdots<t_{N}=t} \sum_{j=1}^{N} \mathcal{D}\left(\mu\left(t_{j-1}\right), \mu\left(t_{j}\right)\right),
$$

and

$$
\left\langle\partial_{f} E(\mu(\tau), f(\tau)), \dot{f}(\tau)\right\rangle=-\int_{D} u_{\mu(\tau), f(\tau)} \dot{f}(t) \mathrm{d} x,
$$

$u_{\mu, f}$ being the solution of

$$
\int_{D} \nabla u \nabla v \mathrm{~d} x+\int_{D} u v \mathrm{~d} \mu=\int_{D} f v \mathrm{~d} x \quad \forall v \in H_{0}^{1}(D) \cap L^{2}(D, \mu),
$$

that is, a weak solution of the problem 2.1]. 
In our numerical implementation, we have chosen two particular dissipation distances, which are introduced below. The first one, denoted by $\mathcal{D}_{\gamma}$, is a dissipation distance related to $\gamma$-convergence. We refer to [6] for comments and remarks concerning the choice of these distances. Let us define $w_{\mu}$ to be the solution of

$$
\left\{\begin{array}{l}
-\Delta w_{\mu}+\mu w_{\mu}=1, \\
w_{\mu} \in H_{0}^{1}(D) \cap L^{2}(D, \mu) .
\end{array}\right.
$$

This function enables us to define a dissipation distance

$$
\mathcal{D}_{\gamma}\left(\mu_{1}, \mu_{2}\right)= \begin{cases}\int_{D}\left|w_{\mu_{1}}-w_{\mu_{2}}\right| \mathrm{d} x & \text { if } \mu_{2} \preceq \mu_{1}, \\ +\infty & \text { if } \mu_{2} \npreceq \mu_{1} .\end{cases}
$$

Definition 2.4 The set of finite measures in $\mathcal{M}_{0}$ is denoted by $\mathcal{M}_{0}^{f}$.

For this case, we have the following (see [6]).

TheOREM 2.5 Let $T>0$ and let $f:[0, T] \rightarrow L^{2}\left(D, \mathbb{R}^{+}\right)$. Let $\mathcal{D}_{\gamma}$ be the irreversible dissipation distance defined as above, and let $\mu_{0} \in \mathcal{M}_{0}$ be an initial condition. Then there exists a generalized minimizing movement $\mu \in \operatorname{GMM}\left(\mathcal{F}, \mathcal{M}_{0}, \mu_{0}\right)$ associated to $\mathcal{F}$ and to the initial condition $\mu_{0}$, where $\mathcal{F}$ is defined by

$$
\mathcal{F}\left(t, \mu_{1}, \mu_{2}\right)=E\left(\mu_{1}, f(t)\right)+\mathcal{D}_{\gamma}\left(\mu_{1}, \mu_{2}\right) .
$$

Moreover, if we assume that the initial condition $\mu_{0}$ is a finite measure in $\mathcal{M}_{0}\left(\mu_{0} \in \mathcal{M}_{0}^{f}\right)$ and that $f \in W^{1, \infty}\left([0, T] ; L^{2}(D)\right)$, then there exists a solution of the rate-independent problem 2.3 - 2.4.

The second dissipation distance occurs if we characterize the state of a debonding membrane by the set $A$ representing the unsticked region. The main difficulty in this situation comes from a relaxation effect: by taking an initial set and an arbitrary distance, the evolution may no longer belong to the family of sets, but to the family of measures. However a delamination model studied in [15] suggests that the dissipation distance is proportional to the surface measure. This matches the choice of a dissipation distance on sets given by

$$
\mathcal{D}_{m}\left(A_{1}, A_{0}\right)= \begin{cases}\left|A_{1} \backslash A_{0}\right| & \text { if } A_{0} \subset A_{1} \text { a.e., } \\ +\infty & \text { if } A_{0} \not \subset A_{1} \text { a.e., }\end{cases}
$$

where $A_{0}$ and $A_{1}$ are sets representing the unsticked area of the membrane. The main result we recall here is that no relaxation process occurs in this case. More precisely, if the initial state is a quasiopen set (i.e. not an arbitrary measure) and with the same model for the energy of the membrane, the solution consists only of shapes, with the identification between the quasi-open set $A$ and the measure $\infty_{D \backslash A}$ defined by

$$
\infty_{D \backslash A}(B)= \begin{cases}0 & \text { if } \operatorname{cap}(B \backslash A)=0, \\ +\infty & \text { otherwise }\end{cases}
$$

Let $\mathcal{A}$ be the family of quasi-open subsets of a bounded design region $D$. A quasi-open set $A$ indicates the region where the membrane is not sticked. We may endow the family $\mathcal{A}$ with $w \gamma$ convergence (see [4, 5] for details): we say that $A_{n} \stackrel{w \gamma}{\longrightarrow} A$ if $w_{A_{n}} \rightarrow w$ weakly in $H_{0}^{1}(D)$ and $A=\{w>0\}$. This convergence is compact and weaker than $\gamma$-convergence.

The dissipation distance $\mathcal{D}_{m}$ defined previously makes sense for all sets $A_{0}$ and $A_{1}$ in $\mathcal{A}$. This dissipation distance is irreversible since we add the condition $A_{0} \subset A_{1}$ a.e. to have a finite distance. 
We consider the functional

$$
\mathcal{F}\left(t, A_{1}, A_{2}\right)=E\left(A_{1}, f(t)\right)+\mathcal{D}_{m}\left(A_{1}, A_{2}\right),
$$

where

$$
E(A, f)=\min \left\{\frac{1}{2} \int_{D}|\nabla u|^{2} \mathrm{~d} x-\int_{D} u f \mathrm{~d} x: u \in H_{0}^{1}(A)\right\} .
$$

The following result is proved in [6]:

Theorem 2.6 Let $T>0$ and let $f:[0, T] \rightarrow L^{2}\left(D, \mathbb{R}^{+}\right)$. Let $A_{0} \in \mathcal{A}$ be an initial quasiopen set and let $\mathcal{A}$ be endowed with $w \gamma$-convergence. Then there exists a generalized minimizing movement $A \in \operatorname{GMM}\left(\mathcal{F}, \mathcal{A}, A_{0}\right)$ associated to $\mathcal{F}$ and to the initial condition $A_{0}$.

If we denote by $\bar{A}(t)$ its lower semicontinuous envelope, that is,

$$
\bar{A}(t)=w \gamma-\lim _{s \uparrow t} A(s)
$$

and assume that $f \in W^{1, \infty}\left([0, T] ; L^{2}(D)\right)$, then the lower semicontinuous envelope $\bar{A}(t)$ of the GMM-solution $(u, A)$ above has the stability property

$$
E(\bar{A}(t), f(t)) \leqslant E(B, f(t))+\mathcal{D}_{m}(B, \bar{A}(t)), \quad \forall t \in[0, T], \forall B \supset \bar{A}(t) \text { q.e. }
$$

REMARK 2.7 In general, we do not know whether the shape flow $t \mapsto \bar{A}(t)$, introduced in Theorem 2.6, satisfies an energy inequality similar to [2.4,

$$
E(\bar{A}(t), f(t))+\operatorname{Diss}_{\mathcal{D}_{m}}(\bar{A},[s, t]) \leqslant E(\bar{A}(s), f(s))+\int_{s}^{t}\left\langle\partial_{f} E(\bar{A}(\tau), f(\tau)), \dot{f}(\tau)\right\rangle \mathrm{d} \tau .
$$

This energy inequality holds upon adding some geometrical a priori constraints on the admissible domains (convexity, exterior cone condition etc.). However, with these a priori constraints, it is not sure if the stability property $(2.13)$ holds.

For more details and analysis we refer the reader to [6].

\section{Debonding 2-state membranes: quasi-open sets}

The choice of the dissipation distance has large influence on the relaxation phenomenon. As stated in Theorem 2.6 the dissipation distance $\mathcal{D}_{m}$ implies no relaxation and produces an evolution where the membrane has only two states: glued or unglued. The unglued region is a quasi-open set.

In a first step, to handle moving domains we have implemented a level set method to deform open sets which is related to the shape derivative of the cost functional $J$.

The main idea is based on representing the domain as the set where a continuous function $\phi$ is positive, and the boundary as the zero level set of $\phi$. The changes made on the boundary are chosen in a direction of descent given by the shape derivative of the cost functional $J$, and are governed by a Hamilton-Jacobi equation.

The functional that we minimize with respect to the shape is

$$
J(A)=-\frac{1}{2} \int_{D} f u_{A} \mathrm{~d} x+|A|=-\frac{1}{2} \int_{D} f u_{A} \mathrm{~d} x+\int_{A} \mathrm{~d} x
$$

where $u_{A}$ is a variational solution of

$$
\left\{\begin{array}{l}
-\Delta u=f \quad \text { in } A \\
u \in H_{0}^{1}(A)
\end{array}\right.
$$


supplemented by the monotonicity constraint $A_{0} \subseteq A$. After computation of the shape gradient, the descent direction is given by

$$
V=-(\nabla u \nabla p+1) \mathbf{n}
$$

where $\mathbf{n}$ is the unit normal vector to the boundary and the function $p$ is the solution of the adjoint problem

$$
\left\{\begin{array}{l}
-\Delta p=-\frac{1}{2} f \quad \text { in } A, \\
p \in H_{0}^{1}(A) .
\end{array}\right.
$$

Notice that the velocity $V$ is defined on all of $D$ and does not require any extension.

The second step consists of a parametrization of $A$ with level sets of a function $\phi_{0}$ such that

$$
\begin{cases}\phi_{0}(x)>0 & \text { if } x \in A, \\ \phi_{0}(x)<0 & \text { if } x \in D \backslash \bar{A}, \\ \phi_{0}(x)=0 & \text { if } x \in \partial A,\end{cases}
$$

for example using the signed distance function to the boundary of $A$.

The deformation is obtained by solving the Hamilton-Jacobi equation

$$
\frac{\mathrm{d} \phi}{\mathrm{d} t}+v|\nabla \phi|=0
$$

with $\phi_{0}$ as initial condition and $v=-(\nabla u \nabla p+1)$. The solution $\phi_{1}$ of (3.4) gives the next open set $A_{1}$ defined by the set where $\phi_{1}$ is positive, after a small time step.

In order to solve the Hamilton-Jacobi equation (3.4) we use the explicit upwind finite difference scheme of Osher and Sethian [19]. The time step, $\Delta t$, between iterations is chosen such that $J\left(A_{1}\right) \leqslant J\left(A_{0}\right)$ and it is limited by the Courant-Friedrichs-Lewy condition

$$
\max _{x \in D} v(x) \Delta t \leqslant \Delta x
$$

where $\Delta x$ is the space discretization step.

The steps of our algorithm are classical:

1. find the function $\phi_{0}$ corresponding to a given set $A_{0}$;

2. compute the velocity $V$ of deformation;

3. compute the solution $\phi_{1}$ of the Hamilton-Jacobi equation and obtain the corresponding domain $A_{1}$, after a small time step. We will deform $\phi_{1}$ with velocity $V$ as long as the corresponding energy is decreasing. We will stop if in ten iterations the energy does not change. In this way we allow some jumps in the evolution of the membrane, but we still do not escape from the local minimum;

4. from time to time reinitialize the function $\phi_{1}$ as the distance function to the set $A_{1}$;

5. put $\phi_{1}$ in the first step and repeat the algorithm.

EXAMPLE 3.1 We assume that the unsticked surface of the membrane at the initial moments is a square from which we remove a rectangle. The force is $f=12 \cdot \chi_{B((0,0), 0.4)}$ and from one iteration to another it increases by 2 .

This local algorithm shows local deformations of the membrane and allows us to see local minimizers rather than global ones. It is not so far from reality, in the sense that it is realistic that a membrane remains in a metastable state before accumulating enough energy to jump the energy 


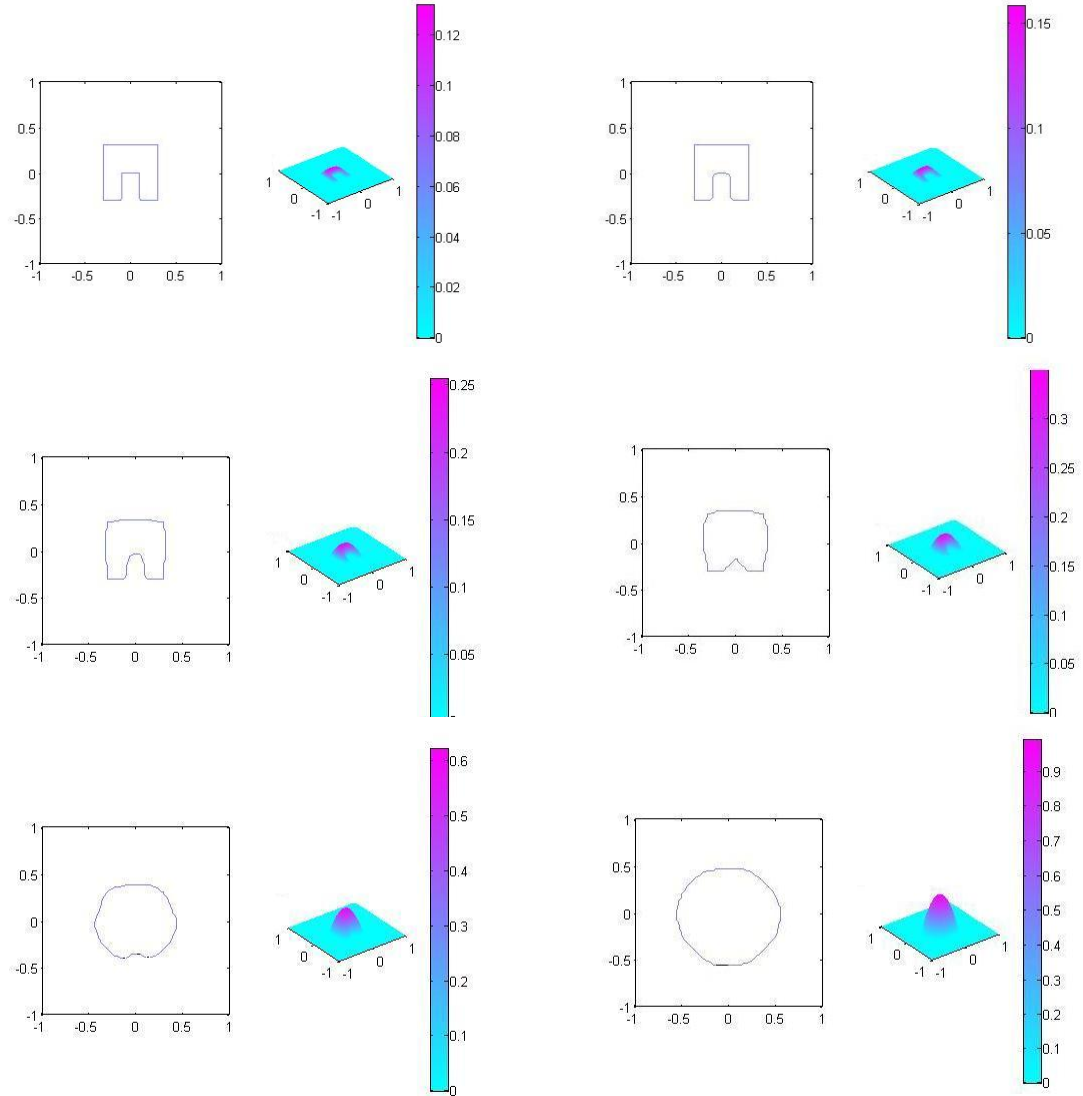

FIG. 1. Local domain evolution.

barriers. Moreover, notice that discrete schemes may stop on local rather than global minimizers because of the lack of convexity of the cost functional.

However in our framework, the model of debonding membranes is based on global minimization; therefore we have implemented an algorithm based on stochastic mechanisms, named an evolutionary strategy. The principle of such algorithms is to make random combinations of open sets to obtain "better" states close to global minimizers for our functional. Therefore, the convergence cannot be proved, but only justified in a probabilistic framework. The main drawback is its cost: all the combinations and tests done in order to decrease the energy are very expensive in terms of computation time.

We will study the case of a circular problem where a jump has to appear. From an analytic point of view this example was discussed in [6]. It is a two-dimensional radially symmetric membrane subjected to an increasing radial force.

Let $D=[0,1] \times[0,1]$ and $A_{0}=\emptyset$, that is, the membrane is initially fully sticked. The force we consider is

$$
f:[0,+\infty) \times D \rightarrow[0,+\infty), \quad f(t, x)=t \cdot \chi_{B(P, 1 / 4)}(x) .
$$

where $P$ is the point $(0.5,0.5)$. 
In order to compute the rate-independent movement, one has to find the solution of

$$
\min _{a \in[0,1]}\left\{E(B(P, a), f(t))+\pi a^{2}\right\} .
$$

The theoretical approach leads to the following results:

- for $0 \leqslant t \leqslant 8 \sqrt{2}$ the functional above is increasing in $a$, hence the minimum is attained at $a=0$ (the membrane remains glued);

- for $8 \sqrt{2} \leqslant t \leqslant 8 \sqrt{2} e^{1 / 4}$, the functional is not increasing in $a$, but the minimum is still reached at $a=0$ (the membrane remains glued);

- for $8 \sqrt{2} e^{1 / 4}<t<16 \sqrt{2}$ the minimum is attained at $a=t /(32 \sqrt{2})$, hence there is a sudden debonding at $t=8 \sqrt{2} e^{1 / 4}$ followed by a continuous debonding up to $t=16 \sqrt{2}$;

- for $t \geqslant 16 \sqrt{2}$ the membrane is fully debonded.

Local algorithms are a priori not able to escape from a local minimum, unlike the algorithm based on evolutionary strategy which relies on the principle of natural evolution: "individuals with a good capacity of adaptation to their environment have a better chance of surviving and reproducing". We do not give here the details of this type of algorithm (for more details we refer the reader to [17], [21]). Our main contribution is the implementation and extension of the parametrization of the family of open sets describing the debonding membrane introduced in [7].

In our computation, the parametrization of the shapes is based on level sets of functions described by Fourier series. Let $\left(a_{m, n}\right)_{(m, n) \in \mathbb{N} \times \mathbb{N}} \subset[-1,1]$ be a family of parameters. We consider the function $k$ defined on $[0,1] \times[0,1]$ by

$$
k(X, Y)=\sum_{m, n} a_{m, n} \sin (\pi n X) \sin (\pi m Y)
$$

where $x=(X, Y)$ is a point of $\mathbb{R}^{2}$. We associate to the set of parameters $\left(a_{m, n}\right)$ the open set

$$
A:=\left\{(X, Y) \in[0,1] \times[0,1]: k(X, Y)<\|k\|_{\infty, D} / 2\right\}
$$

Notice that the boundary of $A$ is, by construction, a union of nonoverlapping simple closed curves (for almost every family of parameters).

In order to respect the monotonicity constraint, a test domain will be $A_{0} \cup A$. We note that in the initial population we also introduce the open set $A_{0}$. A $(\mu, \lambda)$-evolutionary strategy $([21])$ is

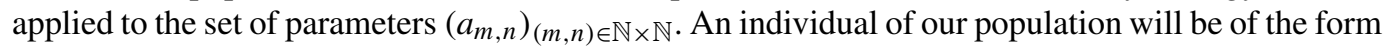
$(Z, \sigma)=\left(\left(z_{1}, \ldots, z_{l}\right),\left(\sigma_{1}, \ldots, \sigma_{l}\right)\right)$, where $\left(z_{1}, \ldots, z_{l}\right)$ are the parameters $\left(a_{m, n}\right)$ after a suitable renumbering and $\left(\sigma_{1}, \ldots, \sigma_{l}\right)$ are the standard deviations used for mutation. In our computation we take $\mu=15, \lambda=105$ and $l=36$.

EXAMPLE 3.2 Consider the position of the membrane totally sticked subjected to the force $f(t, x)=t \cdot \chi_{B(P, 1 / 4)}(x)$. From time 0 to time 15.5 the membrane remains glued. At about time 15.5 the membrane suddenly debonds and the evolution thereafter is a continuous debonding.

REMARK 3.3 The numerical time of the debonding jump (15.5) is close to the theoretical one $\left(8 \sqrt{2} e^{1 / 4}\right)$, and the numerical behaviour is in full agreement with the theoretical expectation. The evolution of the membrane is "almost" independent of the numerical parameters, which means that the numerical time of the debonding jump is stable, remaining in the neighbourhood of $8 \sqrt{2} e^{1 / 4}$ as we modify the initial population or the increasing force step. 

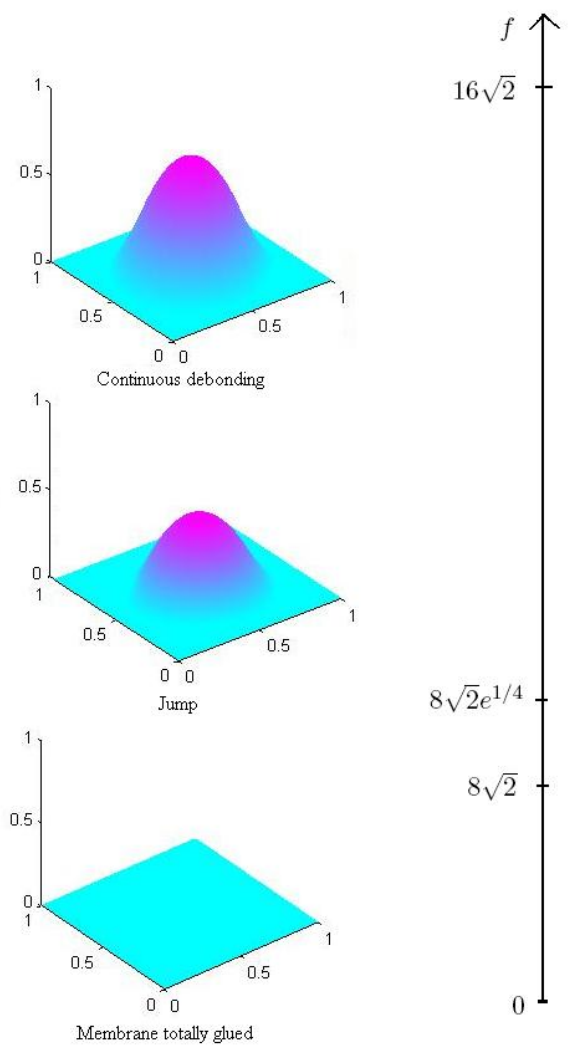

FIG. 2. Global domain evolution.

To compare the two algorithms, local and global, we present the graph of the cost functional at time 15.5 .

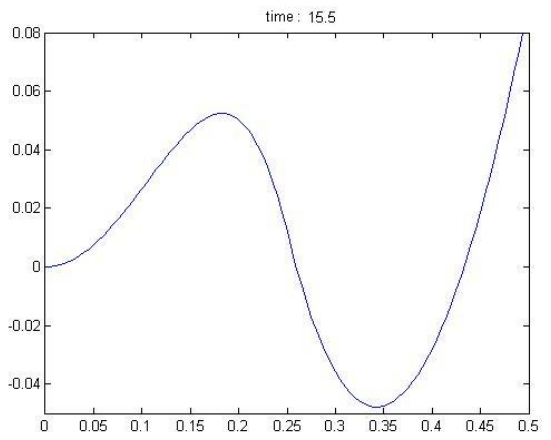

FIG. 3. Cost functional at time 15.5.

Level set method for global minimizers. If we use the algorithm based on the level set method in order to find global minimizers, and we assume that at the initial moment the membrane is 
unsticked on a disc of radius $a=0.05$, then at time 15.5 the minimum is still attained at $a=0.05$. The algorithm cannot escape from the local minimum. If we assume that at the initial moment the membrane is unsticked on a disc of radius $a=0.30$ (i.e. we are close to global minimum), then at time 15.5 the minimum will be attained at $a=0.33$, which is in agreement with the theoretical expectation and also with the global algorithm.
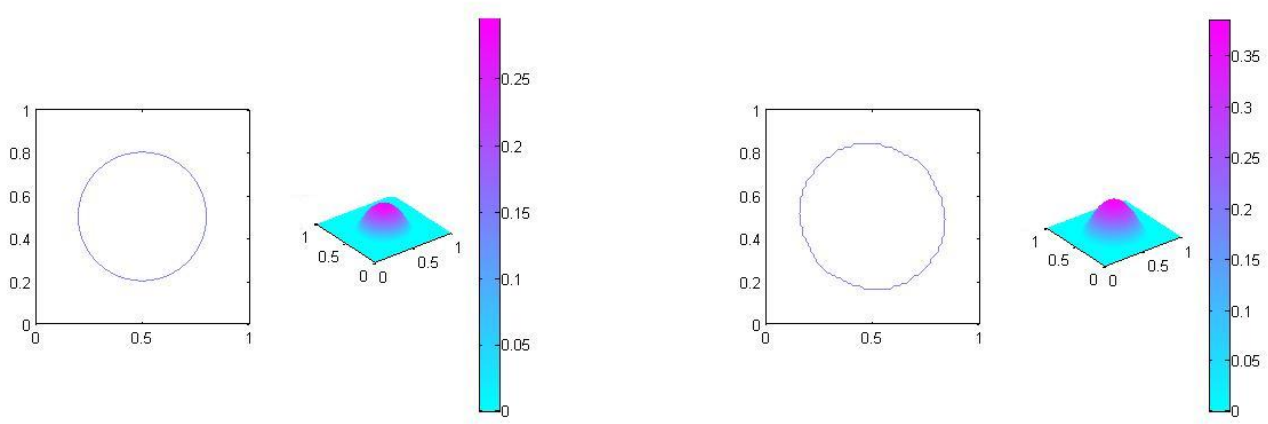

FIG. 4. Position of the membrane at time 14 (left) and at time 15.5 (right).

EXAMPLE 3.4 The initial shape is a crown from which we remove a small part. The force is applied on a slightly larger crown.
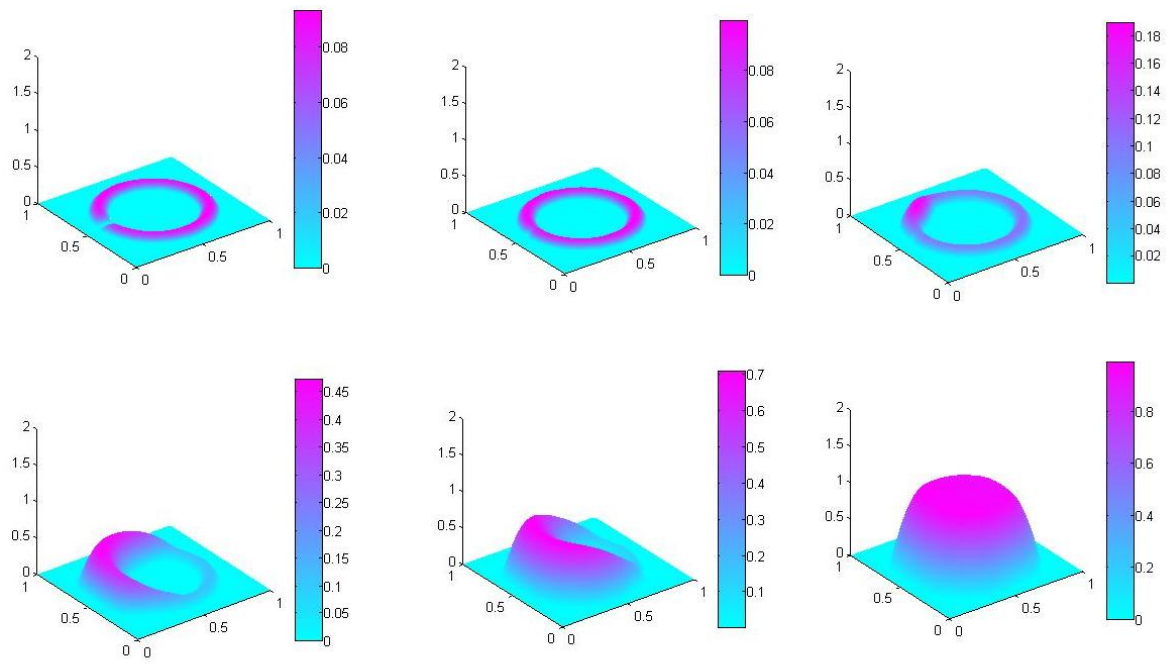

FIG. 5. Global domain evolution.

REMARK 3.5 In this example, the membrane follows the mechanical intuition and debonds the little sticked part of the crown. This shows moreover that the family of simply connected open sets is not stable in this model and the number of connected components of the complement of $A$ may grow. 


\section{Debonding membranes with active fractions of glue: measures}

This section is devoted to the particular case of debonding membranes described by measures $\mu$. Indeed although the evolution of the sticked membrane seems to be only represented by the unsticked region, it may happen that a relaxation process occurs and the membrane may be no more totally sticked or totally debonded but it is sticked with a mixture of glue and free material. Depending on the dissipation distance, one can obtain from an initial domain $A_{0}$ a relaxed solution $\mu_{t}$ related to the density of glue that sticks the membrane. For instance $\mu_{t}(A)=0$ means that the membrane is totally debonded on $A$, whereas $\mu_{t}(A)=+\infty$ corresponds to the perfectly sticked case.

A similar point of view on modelling debonding membranes by a density of glue was developed by Andrews and Shillor [2]; it is a mechanical model based on searching a function $\beta$ governed by an ordinary differential equation taking values between 0 and 1 and representing the percentage of active glue. The main inconvenience of this model is that the equations are defined almost everywhere, which means that the model does not distinguish a membrane sticked on a set of zero Lebesgue measure (for example a segment in two dimensions) but of positive capacity.

All the measures we use for computations are absolutely continuous with respect to the Lebesgue measure. In order to simplify notation, we identify a measure with its density. For numerical implementation the initial measure is a finite one, $\mu_{0}=k_{0}(x) \mathrm{d} x \in \mathcal{M}_{0}^{f}$ (see 2.4 representing the set where the membrane is initially debonded:

$$
k_{0}(x)= \begin{cases}0 & \text { if } x \in A_{0}, \\ 10^{4} & \text { otherwise, }\end{cases}
$$

and the dissipation distance used is $\mathcal{D}_{\gamma}$ (see $(2.7)$ ).

The time interval $[0, T]$ where the function $f$ varies is discretized with a time step $\epsilon$ and we set $t_{k}=\epsilon k, k \in \mathbb{N}$. If the state of the membrane is known at time $t_{k}$, the equilibrium of the membrane at time $t_{k+1}$ is given by the solution of the minimization problem

$$
\min _{\mu \in \mathcal{M}_{0}}\left\{E\left(\mu, f\left(t_{k+1}\right)\right)+\mathcal{D}_{\gamma}\left(\mu\left(t_{k}\right), \mu\right)\right\} .
$$

This is precisely the discrete scheme involved in the general minimizing movement: Theorem 2.5 ensures the convergence, when $\epsilon$ tends to zero, to the general minimizing movement, and in our case, to the rate-independent process.

For numerical experiments, the irreversibility criterion of the $\gamma$-dissipation distance is included in the algorithm, in the class of test measures. Therefore by using the property of the function $w_{\mu}$,

$$
\text { if } \quad \mu_{2} \preceq \mu_{1} \text { then } \mathcal{D}_{\gamma}\left(\mu_{1}, \mu_{2}\right)=\int_{D}\left|w_{\mu_{1}}-w_{\mu_{2}}\right| \mathrm{d} x=\int_{D}\left(w_{\mu_{2}}-w_{\mu_{1}}\right) \mathrm{d} x,
$$

the minimization problem becomes equivalent to

$$
\min _{\mu \in \mathcal{M}_{0}, \mu \leq \mu\left(t_{k}\right)}\left\{E\left(\mu, f\left(t_{k+1}\right)\right)+\int_{D} w_{\mu} \mathrm{d} x\right\}
$$

If we consider the definition of $w_{\mu}$ as the variational solution of 2.6) and take it as a test function, we obtain

The problem becomes

$$
\int_{D} w_{\mu} \mathrm{d} x=\int_{D}\left|\nabla w_{\mu}\right|^{2} \mathrm{~d} x+\int_{D} w_{\mu}^{2} \mathrm{~d} \mu=-2 E(\mu, 1) .
$$

$$
\min _{\mu \in \mathcal{M}_{0}, \mu \leq \mu\left(t_{k}\right)}\left\{E\left(\mu, f\left(t_{k+1}\right)\right)-2 E(\mu, 1)\right\}
$$


From a numerical point of view, we reduce the set $\mathcal{M}_{0}$ by discretizing it on a triangulation in order to consider measures with piecewise constant densities on that triangulation, and we apply a gradient algorithm.

The advantages are:

- the mesh is fixed,

- the problem is reformulated in terms of measures (which have functions as densities), and there is no shape movement, which is more difficult to implement.

The numerical minimization based on a triangulation of the domain $D, \mathcal{T}_{h}=\left(T_{k}\right)_{k=1, \ldots, n}$, relies on the following finite-dimensional spaces:

$$
\begin{aligned}
& X_{h}^{0}:=\left\{v_{h} \in C(D): v_{h} \bigsqcup_{T_{k}} \in \mathbf{P}_{1}, \forall T_{k} \in \mathcal{T}_{h}, v_{h}=0 \text { on } \partial D\right\}, \\
& M_{h}:=\left\{\mu_{h}: \mu_{h}\left\lfloor T_{k}=m_{k} \mathrm{~d} x, m_{k} \in[0, \infty], \forall T_{k} \in \mathcal{T}_{h}\right\},\right.
\end{aligned}
$$

where $\mathbf{P}_{1}$ denotes the space of polynomials in $\mathbb{R}^{2}$ of degree less than or equal to one. Consequently, $X_{h}^{0}$ is a subspace of $H_{0}^{1}(D)$ which contains all the continuous piecewise linear functions on the triangulation $\mathcal{T}_{h}$. Every measure from the class $\mathcal{M}_{0}$ is approximated by a mesure from the class $M_{h}$ whose density is constant on each triangle of $T_{k}$. The numerical unknowns are all the $\left(m_{k}\right)_{k=1, \ldots, n}$. Integration with respect to the discrete measure $\mu_{h}$ has to be understood in the following sense:

$$
\int_{D} f(x) \mathrm{d} \mu_{h}=\sum_{k} m_{k} \int_{T_{k}} f(x) \mathrm{d} x .
$$

The variation of $E(\mu, f)$ with respect to the discrete measure $\mu_{h}$ on each element $T_{k}$ (see [14], [20]) is given in the next theorem.

Theorem 4.1 Let $\mu_{h}=\left(m_{k} \mathrm{~d} x\right)_{k=1}^{n} \in M_{h}$. Then

$$
\frac{\partial E}{\partial m_{k}}=\frac{1}{2} \int_{T_{k}} u_{h}^{2} \mathrm{~d} x \quad \forall k=1, \ldots, n,
$$

where $u_{h}$ is the solution of the discrete version of the problem 2.1 .

We denote by $w_{h}$ the solution of the discrete version of the problem 2.6. The algorithm based on the steepest descent method is:

1. take an initial measure $\mu_{h}^{0} \in M_{h}$ and a number of iterations $I+1$; for $i=0, \ldots, I$ repeat the following steps:

2. calculate $u_{h}^{i}$ and $w_{h}^{i}$ associated to $\mu_{h}^{i}$;

3. calculate the gradient $G^{i}=\left(G_{k}^{i}\right)$, where

$$
G_{k}^{i}=-\frac{1}{2} \int_{T_{k}}\left(u_{h}^{i}\right)^{2} \mathrm{~d} x+\int_{T_{k}}\left(w_{h}^{i}\right)^{2} \mathrm{~d} x \quad \forall k=1, \ldots, n ;
$$

4. determine $\alpha^{i}$ such that

$$
\left.E\left(\left(\mu_{h}^{i}+\alpha^{i} G^{i}\right)^{+}, f\left(t_{k+1}\right)\right)-2 E\left(\left(\mu_{h}^{i}+\alpha^{i} G^{i}\right)^{+}, 1\right)\right) \leqslant E\left(\left(\mu_{h}^{i}, f\left(t_{k}\right)\right)-2 E\left(\left(\mu_{h}^{i}, 1\right)\right)\right.
$$

5. define $\mu_{h}^{i+1}$ by $m_{k}^{i+1}=\left(m_{k}^{i}+\alpha^{i} G_{k}^{i}\right)^{+}$for $k=1, \ldots, n$.

EXAMPLE 4.2 We take the support of the membrane to be $D=[-1,1] \times[-1,1]$, the support of the force acting on the membrane to be the ball $B((0,0), 0.4)$ and the force equal to 10 at the initial moment. At each time iteration the force grows by 2 . The numerical results are presented in Fig. 6. 

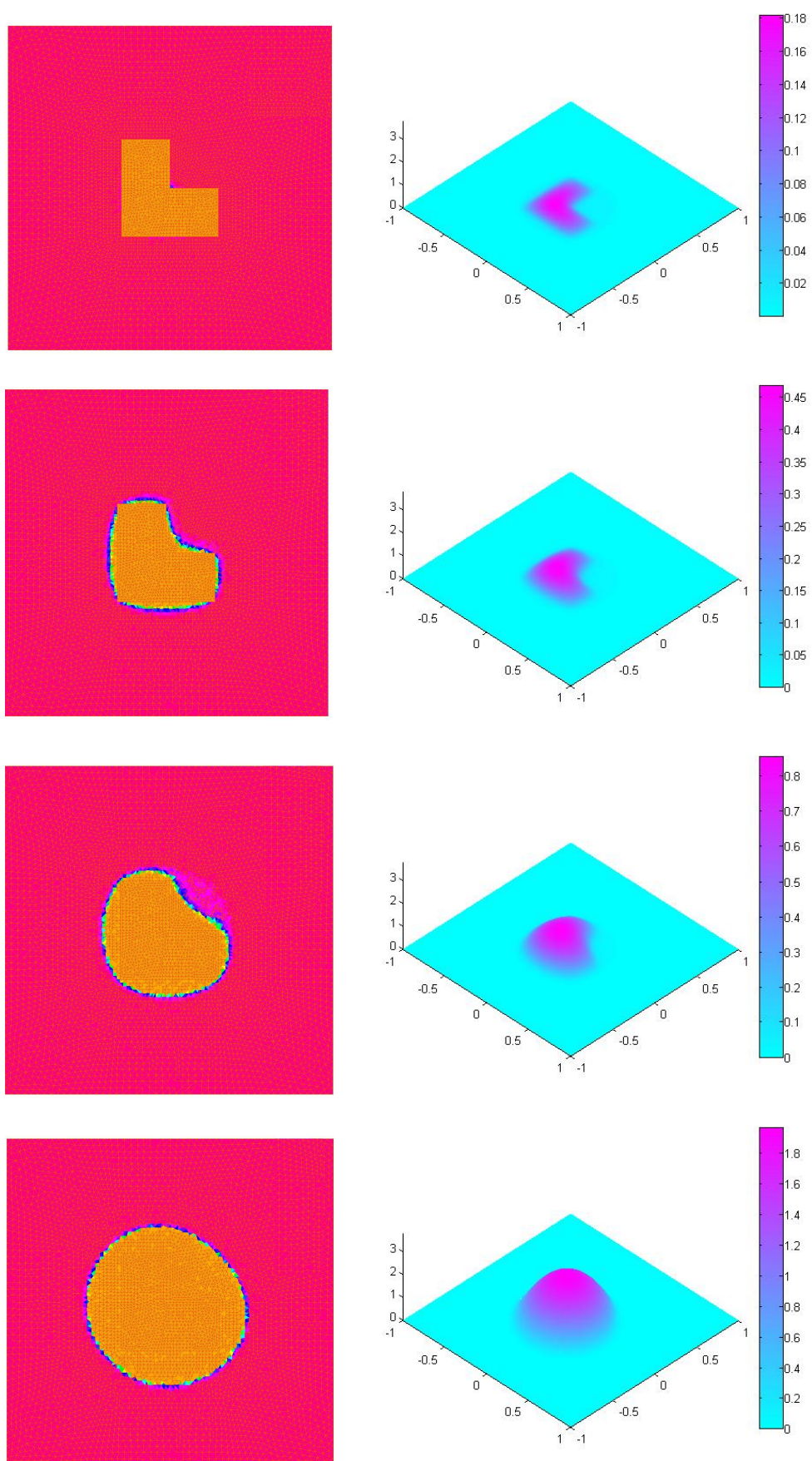

$0 \quad 833$

FIG. 6. Local measure evolution and corresponding positions of the membrane. 
REMARK 4.3 The debonding starts at the entering corner where the gradient is maximal, as expected.

This is a local method. Evolutionary strategies can also be implemented on measures. The main difficulty will be (as for shapes) to provide an efficient method to generate measures.

From the numerical point of view, the minimizing problem will be solved on a smaller set than $\mathcal{M}_{0}^{f}$. We consider only measures which come from a density function generated by Fourier series.
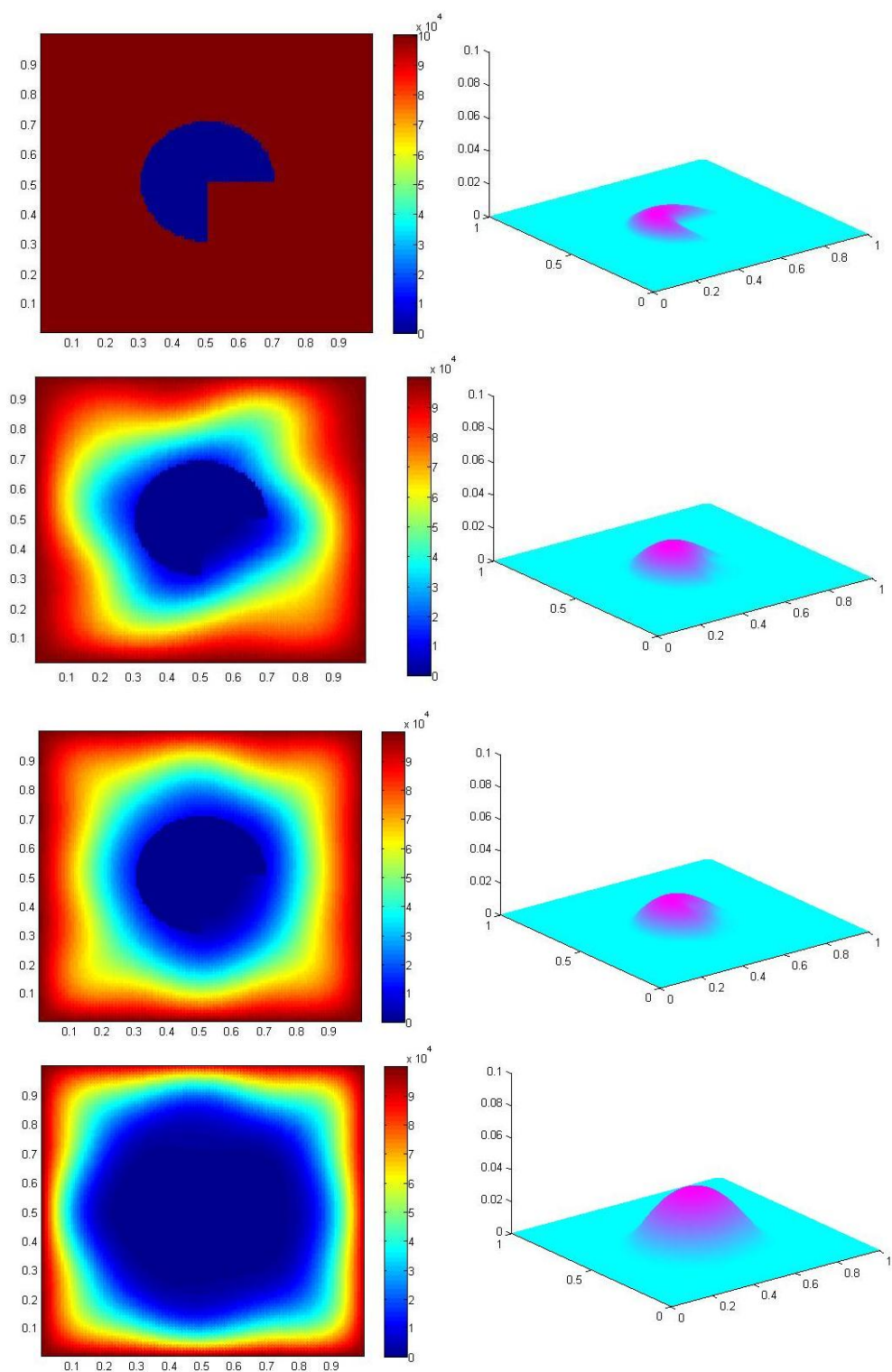

FIG. 7. Global measure evolution. Relaxation process. 
Let $\left(a_{m, n}\right)_{(m, n) \in \mathbb{N} \times \mathbb{N}} \subset[0,1]$ be a family of parameters. We consider the same function $k$ as in 3.5 defined on $[0,1] \times[0,1]$. To generate the measures $\mu \in \mathcal{M}_{0}^{f}(D)$, we first truncate the function $k$ so that its values are in $[0,1]$ :

$$
g(x)=\frac{|k(x)|}{\max _{x \in D}|k(x)|} .
$$

In order to force the generated measure to satisfy the order relation $\mu \preceq \mu_{0}$ we multiply the two truncated functions, $\tilde{g}(x)=g(x) g_{0}(x)$. Then we consider $\mu=10^{5} \tilde{g}(x) \mathrm{d} x$. In this way, we ensure that $\mu \in \mathcal{M}_{0}^{f}$, and $\mu \preceq \mu_{0}$. The genetic operations are done on the set parameters $\left(a_{m, n}\right)$.

EXAMPLE 4.4 Here we assume that the support of the membrane is $D=[0,1] \times[0,1]$. The initial domain is $3 / 4$ of the disc concentric with $D$ of radius 0.2 . We apply a force $f$ on the ball $B((0,0), 0.25)$ which is equal to 0.5 at the beginning, and grows by 0.3 at each step. The evolutionary strategy gives the results presented in Fig. 7.

REMARK 4.5 This numerical example clearly illustrates the relaxation process: the initial form is a simple domain, and the evolution follows in the set of measures.

\section{REFERENCES}

1. Allaire, G., Jouve, F., \& Toader, A.-M. A level-set method for shape optimization. C. R. Math. Acad. Sci. Paris 334 (2002), 1125-1130. Zbl 1115.49306 MR 1911658

2. Andrews, K. T., \& Shillor, M. Dynamic adhesive contact of a membrane. Adv. Math. Sci. Appl. 13 (2003), 343-356. Zbl 1057.74026 MR 2002728

3. BUCUR, D. Regularity of optimal convex shapes. J. Convex Anal. 10 (2003), 501-516. Zbl 1057.49029 MR 2044433

4. Bucur, D., \& Buttazzo, G. Variational Methods in Shape Optimization Problems. Progr. Nonlinear Differential Equations 65, Birkhäuser, Basel (2005). Zbl 1117.49001 MR 2150214

5. Bucur, D., Buttazzo, G., \& Henrot, A. Existence results for some optimal partition problems. $A d v$. Math. Sci. Appl. 8 (1998), 571-579. Zbl 0915.49006 MR 1657219

6. BuCUR, D., Buttazzo G., \& LUX, A. Quasistatic evolution in debonding problems via capacitary methods. Arch. Ration. Mech. Anal. 190 (2008), 281-306. Zbl 1155.74030 MR 2448320

7. BuCUR, D., Durus, I., \& OUdET, E. The eigenvalue problem with conductivity boundary conditions. Control Cybernet. 27 (2008), 811-829. Zbl 1181.35152 MR 2536478

8. Buttazzo, G., \& Dal Maso, G. Shape optimization for Dirichlet problems: relaxed formulation and optimality conditions. Appl. Math. Optim. 23 (1991), 17-49. Zbl 0762.49017| MR 1076053

9. Buttazzo, G., \& Dal Maso, G. An existence result for a class of shape optimization problems. Arch. Ration. Mech. Anal. 122 (1993), 183-195. Zbl 0811.49028 MR 1217590

10. Dal Maso, G. $\Gamma$-convergence and $\mu$-capacities. Ann. Scuola Norm. Sup. Pisa Cl. Sci. 14 (1988), 423464. Zbl 0657.49005 MR 0951228

11. Dal Maso, G., \& Mosco, U. Wiener's criterion and $\Gamma$-convergence. Appl. Math. Optim. 15 (1987), 15-63. Zbl 0644.35033 MR 0866165

12. Dal Maso, G., \& ToAder, R. A model for the quasi-static growth of brittle fractures: existence and approximation results. Arch. Ration. Mech. Anal. 162 (2002), 101-135. Zbl 1042.74002 MR 1897378

13. De Giorgi, E. New problems on minimizing movements. In: Boundary Value Problems for Partial Differential Equations, Res. Notes Appl. Math. 29, Masson, Paris (1993), 81-98. Zbl 0851.35052 MR 1260440

14. FInZI VitA, S. Numerical shape optimization for relaxed Dirichlet problems. Math. Models Methods Appl. Sci. 3 (1993), 19-34. Zbl 0781.49023 MR 1203270 
15. KoČVara, M., Mielke, A., \& RoubíčeK, T. A rate-independent approach to the delamination problem. Math. Mech. Solids 11 (2006), 423-447. Zbl 1133.74038 MR 2245202

16. Mainik, A., \& Mielke, A. Existence results for energetic models for rate-independent systems. Calc. Var. Partial Differential Equations 22 (2005), 73-99. Zbl 1161.74387 MR 2105969

17. MichalewicZ, Z. Genetic Algorithms + Data Structure = Evolution Programs. 3rd ed., Springer, Berlin (1996). Zbl 0841.68047

18. Mielke, A. Analysis of energetic models for rate-independent materials. In: Proc. International Congress of Mathematicians (Beijing, 2002), Vol. III, Higher Ed. Press, Beijing (2002), 817-828. Zbl 1018.74007 MR 1957582

19. Osher, S., \& Sethian, J. Fronts propagating with curvature-dependent speed: algorithms based on Hamilton-Jacobi formulations. J. Comput. Phys. 79 (1988), 12-49. Z Zbl 0659.65132 MR 0965860

20. OUdET, E. Numerical minimization of eigenmodes of a membrane with respect to the domain. ESAIM Control Optim. Calc. Var. 10 (2004), 315-330. Zbl 1076.74045 MR 2084326

21. Schwefel, H.-P. Numerical Optimization of Computer Models. 2nd ed., Wiley, New York (1995).

22. SokoŁowski, J., \& Zolésio, J.-P. Introduction to Shape Optimization. Shape Sensitivity Analysis. Springer Ser. Comput. Math. 16, Springer, Berlin (1992). Zbl 0761.73003 MR 1215733 